\title{
EXPORTACIÓN DE MADERA ASERRADA DE CONÍFERA CHILENA. UN ANÁLISIS DE SU COMPETITIVIDAD
}

\section{CHILEAN EXPORT OF SOFTWOOD SAWNWOOD. AN ANALYSIS ITS COMPETITIVENESS}

\author{
Mauricio Ponce D. ${ }^{1}$, Manuel Contreras G. ${ }^{1}$, Marcia Vásquez $S .^{2}$
}

\begin{abstract}
RESUMEN
Se analiza la competitividad en los principales mercados de las exportaciones chilenas de madera aserrada de pino radiata en el período 1994-2004 (Contreras, 2006). Se aplica la metodología "Competitive Análisis of Nation" (CAN), creada por la Comisión Económica para América Latina y el Caribe (CEPAL), que permite obtener una visión dinámica de las situaciones de competitividad del producto en los mercados estudiados. Los resultados se compararon con un estudio similar del periodo 1987 a 1997, realizado por Fuenzalida (1999). El análisis de la competitividad en el periodo 19942004 muestra que el producto tiene una posición competitiva de Estrella Naciente, en los mercados de China, España, Estados Unidos. y Marruecos; de Estrella Menguante en los mercados de México y Canadá; de Oportunidad Perdida en Japón, Arabia Saudita y Reino Unido; y de Retroceso, en Corea del Sur. Por otro lado, comparando los dos periodos en estudio, se encontró que los mercados de Estados Unidos, Corea del Sur, Japón, Reino Unido y Arabia Saudita, que se repiten en ambos periodos, disminuyeron su posición competitiva del primer periodo al segundo. Asimismo, se puede concluir que el comercio mundial de productos forestales creció más que el comercio de madera aserrada.
\end{abstract}

Palabras Claves: Competitividad, Madera Aserrada de Conífera, Chile.

\begin{abstract}
The competitiveness is analyzed on the principal markets of the Chilean exports of saw wood of radiata pine in the period 1994-2004 (Contreras, 2006). The methodology applied is "Competitive Analysis of Nation" (CAN), created by the Economic Commission for Latin America and the Caribbean (CEPAL), who allows to obtain a dynamic vision of the situations of competitiveness of the product on the studied markets. The results were compared with a similar study of the period 1987 to 1997 realized by Fuenzalida (1999). The analysis of the competitiveness in the period proves to be 1994-2004 that the product has Nascent Start's competitive position, on the markets of China, Spain, United States and Morocco; of Waning Start's position on the markets of Mexico and Canada; of Lost Opportunity in Japan, Saudi Arabia and United Kingdom; and of Setback in South Korea. On the other hand, comparing both periods, one thought that the markets of United States, South Korea, Japan, United Kingdom and Saudi Arabia, which they repeat in both periods, diminished their competitive position of the first period to the second one. Likewise, it is possible to conclude that the world trade of forest products grew more than the trade than saw wood.
\end{abstract}

Keywords: Competitiveness, Conifers Lumber, Chile.

\footnotetext{
${ }^{1}$ Ingenieros Forestales. Facultad de Ciencias Forestales de la Universidad de Talca. Talca, Chile. mponce@utalca.cl

${ }^{2}$ Ingeniero Civil en Industrias Forestales. Facultad de Ciencias Forestales de la Universidad de Talca. Talca, Chile. Autor para correspondencia: mponce@utalca.cl

Recibido: 19 de agosto de 2006. Aceptado: 02 de diciembre de 2006.
} 


\section{INTRODUCCIÓN}

Las ventajas naturales del sector forestal, su capacidad empresarial y las condiciones de mercado permiten competir exitosamente en los mercados internacionales. Sin embargo, ya no es suficiente basar el modelo en las ventajas comparativas, sino se deben crear y mantener ventajas competitivas, que conduzcan hacia la consolidación de los mercados internacionales.

Este estudio contribuye a la comprensión de la competitividad de la madera aserrada chilena en los principales mercados de destino, en el período comprendido entre 1994 a 2004.

En 1974 se inicia un cambio significativo de las estrategias de desarrollo en el país, basada en la plena liberación y apertura hacia el comercio internacional. El sector forestal chileno establece un tipo de desarrollo basado en sus ventajas comparativas y en los beneficios que resulten de la total globalización de sus actividades económicas (Cerda, 1998).

Uno de los factores claves de la competitividad del sector forestal chileno es el bajo costo de formación de madera ${ }^{2}$. Las tasas de crecimiento anuales de las plantaciones de pino radiata fluctúan entre los 20 y $25 \mathrm{~m}^{3} / \mathrm{ha} /$ año, en Nueva Zelanda alcanzan los $20 \mathrm{~m}^{3} / \mathrm{ha} / \mathrm{año}$, en Estados Unidos varían entre 5 y $15 \mathrm{~m}^{3} / \mathrm{ha} / \mathrm{año} \mathrm{y}$ en Escandinavia, no pasa de 2 m³/ha/año (CORMA, 2005).

El desarrollo del sector forestal lleva más de cuatro décadas, presentando un mayor dinamismo en los últimos 20 años. A las ventajas naturales se sumaron la disponibilidad a un bajo costo de superficies aptas para ser plantadas, bajos costos de mano de obra, la proximidad a los puertos, un bajo costo de capital, un ambiente con incentivos a la inversión extranjera, y ventajas competitivas construidas con un esfuerzo conjunto público y privado (Moguillansky y Silva, 2001).

El Decreto Ley $\mathrm{N}^{\circ} 701^{3}$ de fomento forestal de 1974, fue el principal estímulo del sector forestal para acelerar su crecimiento. Se reforzaron las ventajas comparativas, aumentaron las tasas de forestación y actuó en las más diversas fuerzas productivas privadas (Chacón, 1997). Según INFOR (2006), en diciembre del año 2004, se contaba con 2,1 millones de hectáreas de plantaciones forestales, de las cuales aproximadamente 1,4 millones de hectáreas $(67,8 \%)$, corresponde a pino radiata, el $23,6 \%$ a eucalipto, y el 8,6\% restante a otras especies.

Los escenarios globales proyectan una gran competencia de los productos forestales industriales en los próximos 25 años, teniendo relevancia las plantaciones en Argentina, Brasil y Uruguay; como también en Asia, principalmente China e India. Por otro lado, los consumidores prefieren productos y procesos productivos amistosos con el medio ambiente, exigiendo un sector forestal armónico, equilibrado y sustentable (Espejo, 2005).

Las grandes compañías consolidan sus estrategias competitivas en el mercado mundial, estructurando e integrando sus cadenas de valor y, a partir de la mitad de la década de los 90 , asegurando los procesos de internacionalización aprovechando el bajo valor de los activos en los países vecinos, además de inversiones en Estados Unidos y Europa (FAO, 2004).

Según Moguillansky y Silva (op. cit.), la articulación forestal chilena en torno a un mayor valor agregado en los mercados de madera aserrada y remanufacturas, es la que presenta la mayor proyección, porque los mercados de celulosa, astillas y rollizos estarían maduros.

\footnotetext{
${ }^{2}$ Según CORMA (2005), se estima que la materia prima para la elaboración de madera aserrada representa entre el 40 y el $60 \%$ de los costos de producción.

${ }^{3}$ Modificado en 1998 por la Ley 19.561.
} 
La madera aserrada chilena de pino radiata en el mercado internacional, ha podido enfrentarse hasta ahora con una política de precios bajos. Sin embargo, para lograr mejores precios que le permitan mejorar su competitividad, se debe orientar a comercializar productos de mayor valor agregado. (Devlieger y Baettig, 1999).

La preocupación por el deterioro de los recursos naturales ha aumentado, y los consumidores se interesan más por productos que además de cumplir con los estándares de calidad, tengan un bajo impacto negativo en el ambiente (ProChile, 2005) y el principal requerimiento internacional a los productos forestales es la certificación.

Otro aspecto determinante de la competitividad es la biotecnología, que puede cambiar el mapa de competitividad a nivel mundial. Experiencias en Estados Unidos muestran crecimientos superiores a los chilenos en la zona del Sudeste, con coníferas locales. Brasil ha desarrollado un ambicioso programa de genómica de Eucalyptus, con el fin de modificar las variables que permitan aumentar su productividad (CORMA, 2005).

\section{Exportaciones Forestales Chilenas}

En 1994 las exportaciones forestales fueron de Mill. US\$ $1.564 \mathrm{FOB}^{4}$, mientras que en 2004 las alcanzaron los Mill US\$3.397 FOB, cifra récord del sector que significó un incremento de 34,6\% respecto de 2003 y un $47,6 \%$ superior a 2002. Los 10 primeros meses del 2005 llegaron a US\$2.945,1 millones, con un incremento de 5,7\% respecto del año anterior. En 1994 las exportaciones de madera aserrada de pino radiata alcanzó los US\$ 169,2 millones; mientras que en el 2004 se exportó US\$ 569,3 millones, un 42,9\% superior a 2003. El total acumulado en los 10 primeros meses del 2005 alcanzó los US\$ 468 millones, un incremento del 20,8\% respecto de igual período anterior. La madera aserrada de pino radiata participa de un $16 \%$ de las exportaciones sectoriales.

En el año 2004, Estados Unidos fue el mercado más importante para la madera aserrada chilena, que junto a México y Canadá, representaron casi el $60 \%$ del monto total exportado. Le siguen Japón, China y Corea del Sur, que representan aproximadamente un 17\%. En un tercer lugar está Europa, siendo España y Reino Unido los más importantes.

\section{MATERIAL Y MÉTODOS}

\section{Evaluación de la Competitividad}

Se utilizó la metodología propuesta por la Comisión Económica para América Latina (CEPAL), Competitive Analysis of Nations (CAN), que evalúa la competitividad de un producto en sus diferentes mercados. La competitividad se obtiene de la relación existente entre los factores de Atractivo de Mercado y Participación de Mercado, que se expresa como:

$$
\text { Cij }=\mathbf{R}\{\text { Aij; Pij }\}
$$

Donde:

Cij: Competitividad del producto i (madera aserrada), en el mercado objetivo j.

Aij: Atractivo de Mercado para el producto, en el mercado j. Describe la evolución de las importaciones del producto realizadas desde un mercado, resulta del cuociente de los índices Mij y Mj.

Pij: Participación de Mercado del producto en el mercado j. Describe la evolución de la posición del producto, en su nivel de dinamismo en el mercado objetivo, se calcula a través del cuociente de los índices Nij y Mij. 
Mj: Cambios temporales de las importaciones de los productos forestales en su conjunto realizadas por el país j, provenientes del mundo.

Mij: Cambios temporales de las importaciones del producto, realizadas por el país j, provenientes del mundo.

Nij: Cambios temporales en las importaciones del producto, realizadas por el mercado $\mathrm{j}$, provenientes de Chile.

Los datos para calcular los índices Mj y Mij, se obtuvieron desde la Organización de las Naciones Unidas para la Agricultura y la Alimentación, FAO (FAOSTAT, 2006), mientras que para calcular Nij se recurrió a la Oficina de Estudios y Políticas Agrarias (ODEPA).

Los cambios temporales $\mathbf{C i j}$, se obtienen aplicando los promedios de los primeros cinco años de la serie temporal, para la obtención del periodo inicial (denominado A), y los promedios de los últimos cinco años, para el periodo final (denominado B). Este procedimiento se aplica en la obtención de los índices requeridos para calcular los valores críticos, así como también para los índices requeridos en la evaluación de la competitividad (Mj, Mij y Nij).

El análisis utiliza la Matriz de Competitividad (figura 1), que relaciona los factores Atractivo de Mercado y Participación de Mercado, el cual genera cuatro situaciones determinadas por los valores críticos, que entrega la posición competitiva del producto en el mercado seleccionado. Los valores críticos se obtuvieron del índice de crecimiento de las importaciones mundiales de productos forestales y del producto, además del índice de crecimiento de las exportaciones chilenas de madera aserrada hacia el mundo.

El valor crítico del Aij, se obtuvo del cuociente de los índices de crecimiento de las importaciones mundiales del producto y el índice de crecimiento de las importaciones mundiales de productos forestales. El valor crítico de Pij, se obtuvo del cuociente de los índices de crecimiento de las exportaciones chilenas del producto y el índice de crecimiento de las importaciones mundiales del producto.

Para la obtención de los Valores Críticos (V. C.), y de los factores de Atractivo y Participación de Mercado se realiza el siguiente procedimiento, que es idéntico para los tres casos:

$$
\text { V.C. }=(100 \% \pm \text { índice a }) /(100 \% \pm \text { índice } b)
$$

En donde los índices a y b toman los valores correspondientes a cada caso.

\begin{tabular}{|c|c|c|}
\hline \multicolumn{1}{|c|}{$(-)$ Atractivo de Mercado (+) } \\
\cline { 2 - 3 } $\begin{array}{c}(+) \\
\text { Participación } \\
\text { de } \\
\begin{array}{c}\text { Mercado } \\
(-)\end{array}\end{array}$ & $\begin{array}{c}\text { ESTRELLAS } \\
\text { MENGUANTES }\end{array}$ & $\begin{array}{c}\text { ESTRELLAS } \\
\text { NACIENTES }\end{array}$ \\
\cline { 2 - 3 } & RETROCESOS & $\begin{array}{c}\text { OPORTUNIDADES } \\
\text { PERDIDAS }\end{array}$ \\
V.C.: Valor Critico & V.C. \\
\hline
\end{tabular}

Figura 1: Matriz de Competitividad

Fuente: CEPAL (1995). 
Las posiciones competitivas que derivan de la matriz de la Figura se definen como:

a) Estrellas Nacientes: mercados dinámicos donde el producto es competitivo, aumentando su participación.

b) Estrellas Menguantes: mercados estacionarios donde el producto es competitivo, aumentando su participación, donde el mercado está estancado o en declinación.

c) Oportunidades Perdidas: mercados dinámicos en los cuales el producto no es competitivo, disminuyendo su participación.

d) Retrocesos: mercados estacionarios, estancados o en declinación, en los cuales el producto disminuye su participación.

Si un mercado tiene un factor de Atractivo de Mercado(Aij), superior o igual al V. C. será considerado Dinámico, pues posee una estructura importadora favorable. Por el contrario, si es menor al V. C., se considerará Estacionario, es decir, menos atractivo. Los mercados dinámicos se posicionan en la mitad derecha de la matriz.

Si un mercado tiene un factor de Participación de Mercado (Pij), superior o igual al V. C., es considerado Dinámico, pues el producto chileno aumenta o mantiene en el mercado estudiado. Si por el contrario, si el factor fuese menor que el V. C., se considerará Estacionario, es decir, se pierde participación en el mercado. Los mercados dinámicos se posicionan en la mitad superior de la matriz.

\section{Selección de los Mercados}

Se analizaron las exportaciones chilenas del producto según país de destino, durante el periodo comprendido entre 1994 y 2004, disponibles en la Oficina de Estudios y Políticas Agrarias (ODEPA). Como criterio de selección se eligieron los mercados que en la actualidad presentan una participación relevante de las importaciones del producto, y que además cuentan con todos los datos para su evaluación. La base de datos de ODEPA distingue cinco categorías de productos, que se muestran a continuación.

44071011: Tablas aserradas "Shall board", espesor máximo 25 mm; ancho de 100 a 150 mm y largo de 2 a $4 \mathrm{~m}$.

44071019: Las demás maderas aserradas de pino radiata, de espesor superior a $6 \mathrm{~mm}$.

44071090: Las demás maderas aserradas de coníferas, de espesor superior a 6 mm.

44012110: madera en plaquita o partículas, de pino radiata.

44012190: madera en plaquita o partículas, de las demás coníferas.

De las cinco categorías, clasificadas de acuerdo al sistema de códigos arancelarios armonizados de 8 dígitos, sólo las tres primeras se refieren realmente a madera aserrada. De ellas, se considero solo la 44071019, ya que por si sola explica casi la totalidad de las exportaciones totales de madera aserrada de pino radiata realizadas por Chile. A modo de ejemplo, para el periodo que va de enero a diciembre del año 2004, el monto exportado de este producto fue de US\$613.745.533 FOB, mientras que el producto de código 44012190 presentó envíos por tan solo US\$ 1.222.288 FOB, algo así como el $0,2 \%$ del valor anterior. Las restantes categorías se refieren a otras especies de coníferas y otros productos. 


\section{RESULTADOS}

\section{Selección de los Mercados}

Se seleccionaron los mercados de Estados Unidos, México, Japón, España, Arabia Saudita, China, Corea del Sur, Marruecos, Reino Unido y Canadá; que en conjunto representan el 87,7\% de las exportaciones del año 2004 y que habían mantenido un nivel de exportación en tres años anteriores.

\section{Evaluación de la Competitividad}

Cambios temporales (Cij): se obtienen aplicando los promedios de los cinco primeros años de la serie temporal:

$$
\begin{aligned}
& \mathrm{A}=(1994+1995+1996+1997+1998) / 5 \\
& \mathrm{~B}=(2000+2001+2002+2003+2004) / 5 \\
& \mathrm{C}=((\mathrm{B}-\mathrm{A}) / \mathrm{A}) \times 100
\end{aligned}
$$

Este procedimiento se aplica en la obtención de los índices requeridos para calcular los valores críticos, como para los índices en la evaluación de la competitividad (Mj, Mij y Nij).

\section{Determinación de los Valores Críticos:}

a) El valor crítico del factor Atractivo de Mercado Aij, se obtiene del cuociente entre el índice de crecimiento de las importaciones mundiales del producto y el índice de crecimiento de las importaciones mundiales de productos forestales.

Índice de crecimiento de las importaciones mundiales del producto $=-3,70$

Índice de crecimiento de las importaciones mundiales de productos forestales $=12,81$

- Aij: $\mathbf{0 , 8 5}$

Todo mercado en estudio que presente un factor Atractivo de Mercado superior o igual a 0,85 será considerado Dinámico. Si el valor fuese menor, se considerará Estacionario.

b) El valor critico del factor participación de mercado Pij, se obtiene del cuociente entre el índice de crecimiento de las exportaciones chilenas del producto, y el índice de crecimiento de las importaciones mundiales del producto.

Índice de crecimiento de las exportaciones chilenas del producto $\quad=78,41$

Índice de crecimiento de las importaciones mundiales del producto $=-3,70$

- Pij: 1,85

Todo mercado en estudio que presente para el factor Participación de Mercado un valor superior o igual a 1,85, será considerado Dinámico. Si resulta menor, se considerará Estacionario. El valor crítico se considera como el promedio mundial de Participación de Mercado.

Indices de competitividad: se calcularon los índices de competitividad de los factores Atractivo de Mercado y Participación de Mercado, a través de los índices Mj, Mij y Nij, que se presentan a continuación: 
Tabla 1: Índices Mj, Mij y Nij para los mercados en estudio

\begin{tabular}{|l|l|r|r|r|}
\hline $\mathbf{j})$ & Mercado & \multicolumn{1}{l|}{ Mj } & \multicolumn{1}{l|}{ Mij } & \multicolumn{1}{l|}{ Nij } \\
\hline 1 & Estados Unidos & $\mathbf{1 6 , 8 1}$ & 3,09 & 83,01 \\
2 & México & 88,19 & 36,58 & $27.727,04$ \\
$\mathbf{3}$ & Japón & $-28,76$ & $-30,29$ & $-14,16$ \\
4 & España & 29,03 & 47,89 & $1.334,75$ \\
5 & Arabia Saudita & 4,38 & 13,18 & 85,41 \\
6 & China & 48,44 & 35,31 & $31.851,36$ \\
7 & Corea del Sur & $-5,43$ & $-37,46$ & $-66,14$ \\
8 & Marruecos & $\mathbf{1 6 , 2 0}$ & 19,22 & 167,65 \\
9 & Reino Unido & 2,86 & 2,41 & $-42,52$ \\
10 & Canadá & 34,50 & $-10,29$ & $2.234,32$ \\
\hline
\end{tabular}

\section{Obtención del Atractivo de Mercado, Participación de Mercado y Competitividad}

Con los índices requeridos para la evaluación de la competitividad es posible calcular los valores de los factores de Atractivo de Mercado y Participación de Mercado, Aij y Pij, y la posición competitiva resultante para cada mercado.

Tabla 2: Competitividad del producto chileno en los mercados seleccionados

\begin{tabular}{|c|l|c|c|c|}
\hline $\mathbf{j}$ & \multicolumn{1}{|c|}{ Mercado } & $\begin{array}{c}\text { Atractivo de } \\
\text { Mercado (Aij) }\end{array}$ & $\begin{array}{c}\text { Participación de } \\
\text { Mercado (Pij) }\end{array}$ & $\begin{array}{c}\text { Posición Matriz } \\
\text { Competitividad }\end{array}$ \\
\hline $\mathbf{1}$ & Estados Unidos & Dinámico & Dinámico & Estrella Naciente \\
\hline $\mathbf{2}$ & México & Estacionario & Dinámico & Estrella Menguante \\
\hline $\mathbf{3}$ & Japón & Dinámico & Estacionario & Oportunidad Perdida \\
\hline $\mathbf{4}$ & España & Dinámico & Dinámico & Estrella Naciente \\
\hline $\mathbf{5}$ & Arabia Saudita & Dinámico & Estacionario & Oportunidad Perdida \\
\hline $\mathbf{6}$ & China & Dinámico & Dinámico & Estrella Naciente \\
\hline $\mathbf{7}$ & Corea del Sur & Estacionario & Estacionario & Retroceso \\
\hline $\mathbf{8}$ & Marruecos & Dinámico & Dinámico & Estrella Naciente \\
\hline $\mathbf{9}$ & Reino Unido & Dinámico & Fstacionario & Oportunidad Perdida \\
$\mathbf{1 0}$ & Canadá & Estacionario & Dinámico & Estrella Menguante \\
\hline
\end{tabular}


La información anterior se presenta de manera gráfica, de acuerdo a su posición en la Matriz de Competitividad, de la Figura 2. En este plano cartesiano, el eje de las abscisas (eje x), representa al factor de Atractivo de Mercado, mientras que el eje de las ordenadas (eje y), representa al factor de Participación de Mercado, en donde el eje X toma el valor 1,85 (valor crítico de Participación de Mercado), y el eje Y toma el valor 0,85 (valor crítico de Atractivo de Mercado).

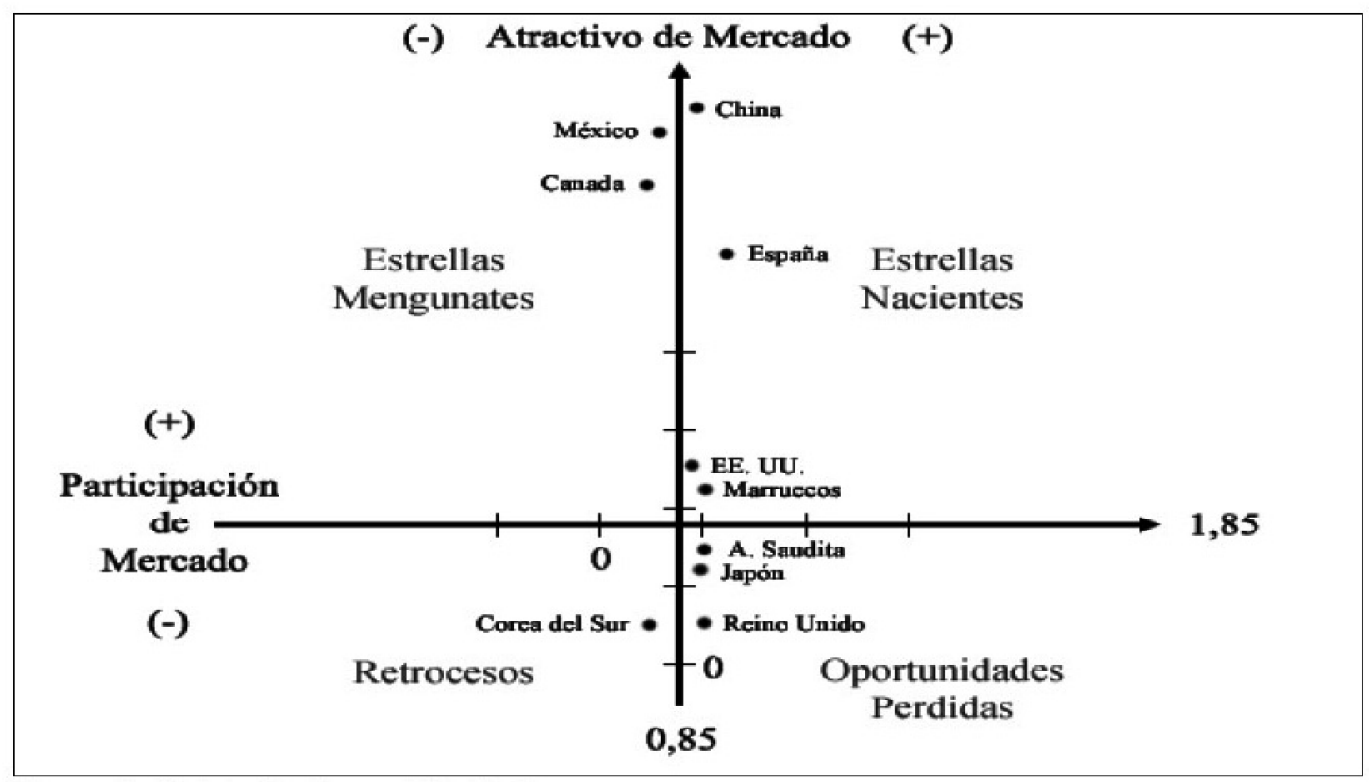

Figura 2: Matriz de Competitividad

En las siguientes tablas se muestra la posición competitiva obtenida en el estudio realizado entre los años 1987-1997, y la comparación con los datos que se entregan en este estudio en aquellos mercados que son coincidentes. 
Tabla 3: Posiciones competitivas, periodo 1987-1997

\begin{tabular}{|c|c|c|}
\hline & Mercado & Posición Competitiva \\
\hline $\mathbf{1}$ & Estados Unidos & Estrella Naciente \\
\hline $\mathbf{2}$ & Reino Unido & Estrella Menguante \\
\hline $\mathbf{3}$ & Arabia Saudita & Oportunidad Perdida \\
\hline $\mathbf{4}$ & Emiratos Arabes & Oportunidad Perdida \\
\hline $\mathbf{5}$ & Japón & Estrella Naciente \\
\hline $\mathbf{6}$ & Egipto & Oportunidad Perdida \\
\hline $\mathbf{7}$ & Rep. Dominicana & Oportunidad Perdida \\
\hline $\mathbf{8}$ & Argentina & Retroceso \\
\hline $\mathbf{9}$ & Corea del Sur & Estrella Naciente \\
\hline
\end{tabular}

Fuente: Fuenzalida (1999).

Tabla 4: Evolución de las posiciones competitivas

\begin{tabular}{|l|c|c|}
\hline \multicolumn{1}{|c|}{ Mercado } & \multicolumn{2}{|c|}{ Periodo } \\
\cline { 2 - 3 } & $1987-1997$ & $1994-2004$ \\
\hline EE.UU. & Estrella Naciente & Estrella Naciente \\
\hline Reino Unido & Estrella Menguante & Op. Perdida \\
\hline Arabia Saudita & Op. Perdida & Op. Perdida \\
\hline Japón & Estrella Naciente & Op. Perdida \\
\hline Corea del Sur & Estrella Naciente & Retroceso \\
\hline
\end{tabular}

En la tabla se observa que el producto conservó su posición competitiva en los mercados de EE.UU. y Arabia Saudita, mientras que en Japón, Corea del Sur y Reino Unido cambió de posición. Sin embargo, en todos ellos existieron variaciones en los niveles de Atractivo y Participación de Mercado, entre un período y otro. La situación competitiva de estos cinco mercados, para ambos períodos, se muestra de manera gráfica en la figura 3 .

Los dos planos cartesianos que se aprecian en esta figura 3 representan los dos períodos estudiados para el producto. Para del período 1987-1997 los V. C. son 0,93 y 1,05 para Participación de Mercado y Atractivo de Mercado respectivamente, mientras que la otra corresponde al período 1994-2004. Con línea punteada se muestra la evolución de ambos periodos. 


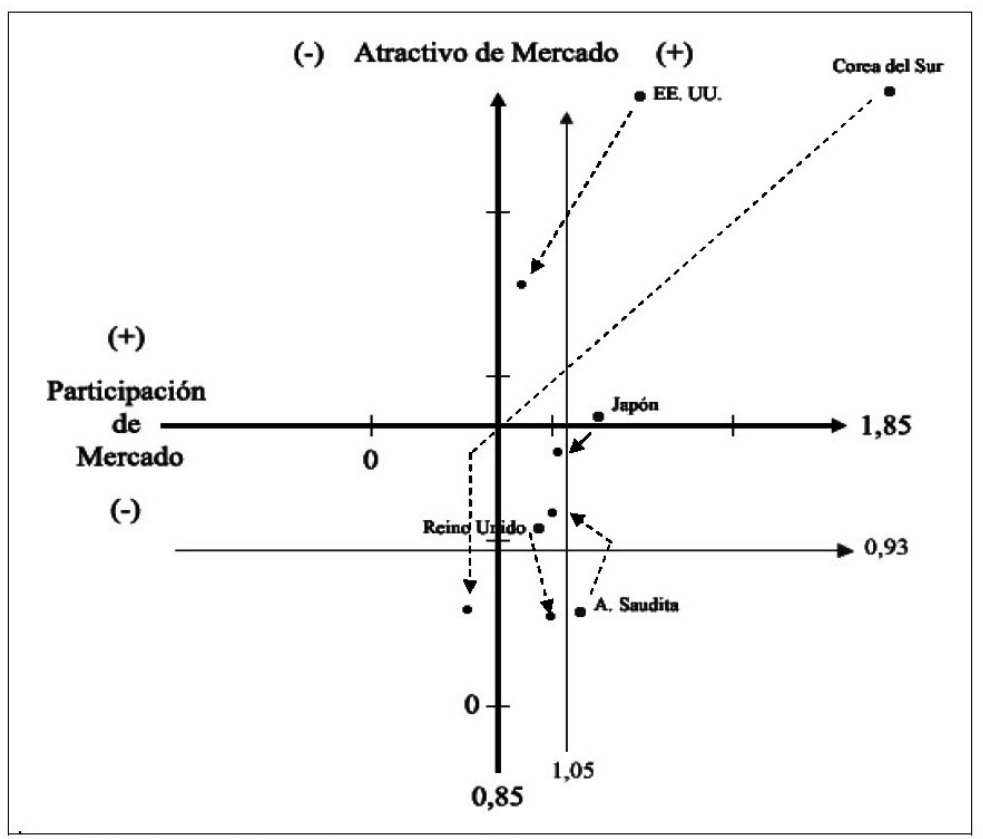

Figura 3: Evolución de las posiciones competitivas

\section{DISCUSIÓN}

Con relación a la periodo 1994-2004, en la figura 2 se aprecia que el nivel de Atractivo de Mercado para la madera aserrada de coníferas fue relativamente similar en todos los países estudiados, con valores que se concentraron en torno al promedio mundial o valor critico $(0,85)$. A pesar de esto, siete de diez mercados presentaron un índice de Atractivo de Mercado superior al valor crítico, es decir, fueron Dinámicos.

El mercado más Dinámico fue España (A4= 1,15), seguido por Arabia Saudita (A5=1,08), Marruecos (A8=1,03), Reino Unido (A9=1,00), Japón (A3=0,98), China (A6=0,91), y EE.UU. $(\mathrm{A} 1=0,88)$. En seis mercados las importaciones de productos forestales y de madera aserrada presentaron índices positivos de crecimiento, y sólo en Japón fue negativo.

Por otra parte, el mercado más Estacionario fue Corea del Sur $(A 7=0,66)$, seguido por Canadá $(\mathrm{A} 10=0,67)$, y México $(\mathrm{A} 2=0,73)$. Este último presentó altos índices de crecimiento en sus importaciones de productos forestales (Mj), y de madera aserrada (Mij), sin embargo, la relación existente entre ambos ( $\mathrm{Mij} / \mathrm{Mj}$ ), fue inferior al promedio mundial.

Los mercados de comportamiento Dinámico fueron China $(P 6=233,85)$, México $(P 2=201,68)$, Canadá (P10= 26,03), España (P4= 9,70), EE.UU. (P1= 2,57), y Marruecos ( $\mathrm{P} 8=2,25)$. Los mercados en los que el producto perdió participación, en relación al promedio mundial fueron Corea del Sur $(\mathrm{P} 7=0,54)$, Reino Unido (P9= 0,56), Japón (P3= 1,23), y Arabia saudita (P5= 1,64). 


\section{Evolución de la Competitividad}

Al analizar de forma comparativa los resultados obtenidos por Fuenzalida (1999), que evaluó la competitividad de las exportaciones de madera aserrada de coníferas en nueve principales mercados de destino entre los años 1987 y 1997; se observa que gran parte de éstos se mantienen dentro de los primeros lugares de importancia como destino de las exportaciones del producto. Se destaca la posición obtenida por EE.UU., ya que durante los dos períodos, se mantuvo como el principal destino de las exportaciones. Cinco son los mercados que se repiten en ambos análisis.

La madera aserrada de pino radiata se encuentra actualmente en una posición competitiva a nivel mundial de Estrella Menguante, ya que la evolución de los valores críticos (de 1,05 a 0,85), muestra que el producto es Dinámico en un mercado mundial que ha disminuido sus niveles de atractivo, ganando participación de mercado (de 0,93 a 1,85).

La caída en el nivel de Atractivo de Mercado mundial, se debe a la lenta recuperación de mercado mundial de la madera aserrada de conífera, posterior a la crisis asiática, en relación a la rápida recuperación del mercado mundial de los productos forestales.

En EE. UU., el producto se mantiene en una posición competitiva de Estrella Naciente en ambos períodos. El movimiento de las posiciones competitivas muestra una disminución del Atractivo de Mercado, y también de Participación de Mercado. Sin embargo, esta aparente pérdida de competitividad se debe al efecto del elevado valor del índice $\mathrm{Nij}(1.565,90 \%)$ durante el período 1987-1997. Si se calcula la cuota de mercado para los años 1997 y 2004, se puede apreciar que fue un aumento significativo entre un período y otro, pasando de un $1,18 \%$ de participación en el año 1997 , al 3,11\% en el año 2004.

En Reino Unido la madera aserrada de pino radiata pasó de la posición de Estrella Menguante a Oportunidad Perdida. El aumento en el nivel de Atractivo de Mercado, en comparación con el primer período, se debe a que la demanda por productos forestales y madera aserrada creció lentamente entre los años 1994 a 2004 (2,86\% y 2,41\% respectivamente), en comparación a los índices de crecimiento del período 1987-1997. Sin embargo, el producto se mostró poco competitivo al no poder sostener el crecimiento del mercado, perdiendo participación en aproximadamente un 50\%.

Por su parte, en Arabia Saudita la madera aserrada se mantuvo durante los dos períodos como una Oportunidad Perdida. El Atractivo de Mercado disminuyó dentro del segundo período; sin embargo, la Participación de Mercado aumentó en 2,78 veces.

En el caso de Japón, el producto mantuvo una posición competitiva de Estrella Naciente, durante el período 1987-1997, alcanzando elevados índices de crecimiento (259,58\%), en un mercado de crecimiento para los productos forestales $(46,55 \%)$, y especialmente la madera aserrada $(93,36 \%)$. Sin embargo, tras la crisis asiática, el mercado disminuyó los niveles de importación de estos productos. En este escenario la madera aserrada chilena perdió participación de mercado, entre ambos períodos en un $33,88 \%$.

El mercado de Corea del Sur durante el período 1987-1997, se encontraba en pleno desarrollo, presentando un elevado Atractivo de Mercado. El producto chileno se encontraba en una buena posición de competitividad, abarcando el 15,18\% del mercado de la madera aserrada. Luego de la crisis, Corea del Sur se transformó en el mercado en que Chile evidenció la mayor pérdida de competitividad, que provocó una importante caída en la Participación de Mercado 


\section{CONCLUSIONES}

En los últimos 18 años, correspondientes al período 1987-2004, los mercados de Estados Unidos, Reino Unido, Japón, Arabia Saudita, Corea del Sur y Emiratos Arabes, fueron de manera ininterrumpida los principales destinos de las exportaciones chilenas de madera aserrada. En el año 2004, los diez principales mercados de destino de las exportaciones chilenas de madera aserrada, representaron aproximadamente el 93,43\% del monto total exportado. De todos ellos, los mercados de Estados Unidos, México y Japón representaron el 70\% del total. A pesar de esta tendencia a concentrar gran parte de las exportaciones en pocos mercados, quedó demostrada la capacidad empresarial del sector forestal chileno, al saber diversificar a tiempo sus mercados, en especial en períodos de crisis internacional.

En el periodo 1994-2004, las exportaciones chilenas de madera aserrada a los mercados de EE.UU., China, España y Marruecos, son clasificadas como Estrellas Nacientes, siendo altamente competitivo, sosteniendo el crecimiento en los respectivos mercados.

En los mercados de México y Canadá, la madera aserrada chilena alcanzó la posición de Estrella Menguante, en los cuales el producto chileno fue competitivo, ganando participación de mercado.

Los mercados de Japón, Arabia Saudita y Reino Unido se clasificaron como Oportunidades Perdidas, mostrándose el producto chileno poco competitivo. De seguir mejorando la Participación de Mercado en Arabia Saudita, podría evolucionar a una posición de Estrella Naciente, para lo cual se deben mejorar las estrategias comerciales con ese país.

El único mercado donde el producto chileno se encuentra en una posición de Retroceso fue Corea del Sur, ya que los niveles de Atractivo de Mercado tras la crisis asiática se redujeron, perdiéndose Participación de Mercado durante el período en un $46 \%$.

Por otro lado, comparando la evolución de la competitividad entre los períodos 1987-1997 y 19942004, demostró que el Atractivo de Mercado mundial disminuyó en el segundo período, mientras que la Participación de Mercado del producto chileno a nivel mundial aumentó. Los principales cambios ocurrieron en los mercados de Arabia Saudita, que aumentó su Participación de Mercado cercano a la posición de Estrella Naciente; y en el mercado de Corea del Sur, que pasó drásticamente de una posición de Estrella Naciente a la posición de Retroceso.

\section{BIBLIOGRAFÍA}

CHACÓN, I. 1997. Evaluación económica del DL 701 en la VII Región. Serie Estudios No 1 .Talca, Chile. Universidad de Talca. 99 p.

CERDA, I. 1998. Trayectoria del sector forestal chileno: sus logros y desafíos. Informe técnico $\mathrm{N}^{\circ}$ 140. Santiago, Chile. INFOR. 173 p.

COMISIÓN ECONÓMICA PARA AMÉRICA LATINA Y EL CARIBE (CEPAL). 1995. Análisis de la Competitividad de las Naciones (CAN). Programa computacional para evaluar y describir el medio competitivo internacional. Santiago, Chile. 72 p.

CONTRERAS, M. 2006. Competitividad de las exportaciones chilenas de madera aserrada de pino radiata en sus principales mercados. Memoria para optar al titulo de Ingeniero Forestal. Universidad de Talca. $62 \mathrm{p}$.

CORMA. 2005. Biotecnología forestal, llave de la competitividad futura (en línea). Santiago, Chile. Disponible en http://www.corma.cl/publicaciones/mailing_ejecutivo/A9-N2.html. Consultado 10 feb. 2006. 
DEVLIEGER, F.; BAETTIG, R. 1999. Ingeniería de aserraderos: fundamentos de planificación y gestión. Serie Técnica N ${ }^{\circ}$. Universidad de Talca. 144 p.

ESPEJO, M. 2004. Un futuro lleno de desafíos. En: Chile Forestal № 308:1.

FAO. 2004. Informe nacional Chile: estudio de tendencias y perspectivas del Sector Forestal en América Latina Documento de Trabajo (en línea). Roma, Italia. Disponible en http://www.fao.org/documents/show_cdr.asp?url_file=/docrep/007/j2576s/j2576s06.htm. Consultado 5 feb. 2006.

FAOSTAT. 2006. Base de datos estadísticos FAOSTAT (en línea). Roma, Italia. Disponible en http://faostat.fao.org/faostat/collections?subset=forestry. Consultado 10 mar. 2006.

FUENZALIDA, G. 1999. Competitividad de la madera aserrada de confieras de exportación: El caso de la madera aserrada de pino radiata chileno. Memoria para optar al titulo de Ingeniero Forestal. Universidad de Talca. $51 \mathrm{p}$.

INFOR. 2005a. Exportaciones forestales chilenas: enero-diciembre 2004. Boletín estadístico $\mathrm{N}^{\circ}$ 99. Santiago, Chile. INFOR. 100 p.

INFOR. 2005b. Estudio de disponibilidad de pino radiata: 2003-2032 (en línea). Santiago, Chile Disponible en http://www.infor.cl/webinfor/publicaciones/Documentos_2005/disponibilidad_marzo05.pdf. Consultado 5 feb. 2006.

INFOR. 2006. Estadísticas del sector forestal (en línea). Santiago, Chile. Disponible en http// www.infor.cl. Consultado 24 ene. 2006.

MOGUILLANSKI, G.; SILVA, V. 2001. Estrategias empresariales y políticas públicas: el futuro del complejo forestal en Chile. En: Gomá, O. Más allá del bosque: transformar el modelo exportador. Santiago, Chile. Editorial FLACSO. pp. 107-144.

ODEPA. 2005. Inserción de la agricultura chilena en los mercados internacionales (en línea). Santiago, Chile. Disponible en http://www.prochile.cl/documentos/est_economi cos/odepa_insercion_dic_05.pdf. Consultado 3 feb. 2006.

DEPA. 2006. Base de datos de comercio exterior, avance producto país (en línea). Santiago, Chile. Disponible en http://www.odepa.cl. Consultado 3 feb. 2006.

PORTER, M. 1991. Ventaja Competitiva de las Naciones. Segunda edición. Editorial Vergara. $1025 \mathrm{p}$.

PROCHILE. 2003. VI Rueda internacional de negocios: programa formación de exportadores. Talca, Chile. 1 disco compacto.

PROCHILE. 2005. Certificación forestal en Chile avanzando hacia la sustentabilidad (en línea). Santiago, chile. Disponible en http://www.prochile.cl/documentos/forestal01.php. Consultado 25 ene. 2006. 
